\title{
AN OVERVIEW OF THE SAFSIM COMPUTER PROGRAM
}

\author{
Dean Dobranich \\ Sandia National Laboratories \\ Nuclear Technology Department 6513 \\ Albuquerque, NM 87185 \\ 505-845-7014
}

$\operatorname{con} 9-90 / 03 \cdots-42$

\section{Abstract}

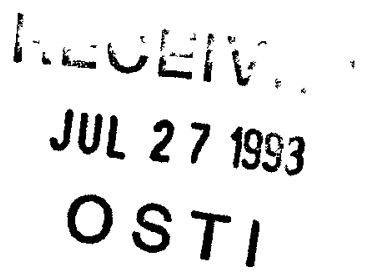

SAFSIM (System Analysis Flow SIMulator) is a FORTRAN computer program that provides engineering simulations of user-specified flow networks at the system level. It includes fluid mechanics, heat transfer, and reactor dynamics capabilities. SAFSIM provides sufficient versatility to allow the simulation of almost any flow system, from a backyard sprinkler system to a clustered nuclear reactor propulsion system. In addition to versatility, speed and robustness are primary goals of SAFSIM development. The current capabilities of SAFSIM are summarized and some sample applications are presented.

\section{INTRODUCTION}

The key feature of a fluid mechanics systems level computer program is the ability to simulate the integrated performance of all components of a system. Thus all the potentially complex interactions and feedbacks between components can be captured. In such simulations it is usually sufficient to model the components in a 1-D or lumped fashion. Such systems level programs have been developed and used with great success in the light-water reactor industry for many years (for example, see Ransom, 1981). However, these computer programs contain a significant amount of capability particular to light-water reactors and therefore are not truly general purpose.

The motivation for SAFSIM (Dobranich, 1992) development is the desire to have a generalpurpose computational tool to provide quick and inexpensive engineering performance simulations of complicated user-specified systems. The simulations are intended to provide a first-look understanding of a systems steady-state and transient behavior during operational and off-normal conditions. It is also desired that this tool have the ability to accommodate changes in the problem definition via changes in an input file rather than changes in the computer program. Thus, all geometric and operational information is provided by the analyst, allowing the analyst to select a level of modeling detail consistent with the problem detail available. SAFSIMs network approach allows the user to "build" a geometric representation of any system by appropriately coupling networks of fluid flow and heat transfer finite elements.

\section{PROGRAM DESCRIPTION}

SAFSIM currently contains three basic physics modules: (1) fluid mechanics, (2) structure heat transfer, and (3) reactor dynamics. All three modules are coupled to allow the prediction of system performance. The analyst can employ any or all of the physics modules as the problem dictates.

\section{Fluid Mechanics}

The fluid mechanics module solves the single-phase conservation of mass, momentum, and energy equations and is based on a 1-D finite element model. The module allows the specification of a userdefined flow network or multiple networks. The network approach allows finite elements to be connected in series or parallel; thus pseudo multidimensional representations of components can be constructed. The fluid can be either a gas or a liquid; also, mixing models are included that allow the specification of multiple gases. Either open or closed networks can be modeled. The fluid mechanics module includes the solution of the compressible or incompressible mechanical and thermal energy equations. The analyst can select a quasi-static or fully implicit dynamic solution of the fluid mechanics equations. For the quasi-static solution, only the boundary conditions and loads

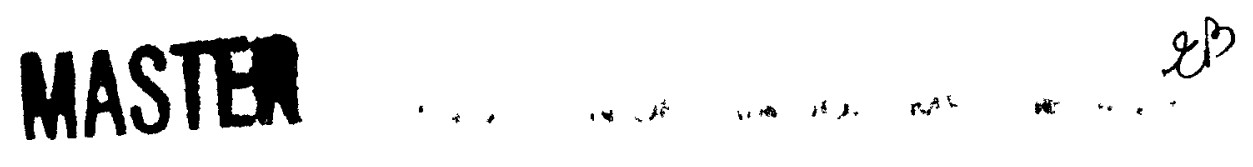




\section{DISCLAIMER}

This report was prepared as an account of work sponsored by an agency of the United States Government. Neither the United States Government nor any agency Thereof, nor any of their employees, makes any warranty, express or implied, or assumes any legal liability or responsibility for the accuracy, completeness, or usefulness of any information, apparatus, product, or process disclosed, or represents that its use would not infringe privately owned rights. Reference herein to any specific commercial product, process, or service by trade name, trademark, manufacturer, or otherwise does not necessarily constitute or imply its endorsement, recommendation, or favoring by the United States Government or any agency thereof. The views and opinions of authors expressed herein do not necessarily state or reflect those of the United States Government or any agency thereof. 


\section{DISCLAIMER}

Portions of this document may be illegible in electronic image products. Images are produced from the best available original document. 
(such as convection from a structure) are time dependent; the fluid temperature, mass flow rate, and density time-derivative terms are neglected. For the dynamic solution option, the fluid temperature and mass flow rate time-derivative terms are included. However, the density timederivative term (mass accumulation) in the mass continuity equation is omitted in the current version. Also, an option exists to bypass the mechanical energy equation solution during problem execution if desired to speed execution. Although the mechanical and thermal energy equations are solved separately, they are iteratively coupled to provide the solution to a total energy equation for the fluid. Super element capability is provided for the mechanical energy equation solution. Super elements allow a series of finite elements to be grouped into one equivalent super element, greatly improving computational efficiency.

Advection of, conduction in, and convection to the fluid are modeled in the thermal energy equation. Upwind finite elements are employed for the thermal energy equation with automatic determination of the optimum upwind factor based on the Peclet number. Convective heat transfer from any solid structure to the fluid is based on the log-mean temperature difference, thus improving accuracy. More than one convective surface can be coupled to any fluid mechanics element.

An ideal equation of state is used for gases to specify density as a function of temperature and pressure. For liquids, the density is specified as a function of temperature only. Fluid conductivity and specific heat are specified by third-order polynomial equations. Either a power-law or Sutherland-law equation can be used to specify fluid viscosity. Multiple temperature ranges can be specified for the three fluid properties. A user-supplied equation of state and property interface is also available. Different time steps can be specified for each fluid mechanics flow network. Pressure or mass flow rate boundary conditions can be applied to any node of a flow network for the mechanical energy equation. Temperature or zero heat flux boundary conditions can be applied to any node for the thermal energy equation. Mass fraction boundary conditions for each gas of a multiple gas network can also be specified for any node.

Four special finite elements are included to increase modeling versatility: (1) a porous media element, (2) a choked flow boundary element, (3) a compressor/pump element, and (4) a distributed flow manifold element. The porous media element allows the user to specify an element porosity to simulate porous media. The analyst may select the Ergun, Achenbach, or modified-Ergun correlations for calculation of the porous media friction factor. If none of these correlations is adequate, analysts can define their own via the input file. Fluid conductivity enhancement due to dispersive effects is included. A choked flow boundary element allows the simulation of choked flow based on solution of the compressible, isentropic flow equations. Discharge coefficients can be added to this finite element to account for any flow inefficiencies. The compressor/pump finite element requires the input of a pump characteristic curve along with rated and operational speeds. The pump characteristic curve is adjusted for varying operational speeds based on the pump similarity laws. A distributed flow manifold finite element accounts for the fact that flow can enter or exit this element along its entire length and not just at the nodes. Also, transpiration flow effects on the friction factor and heat transfer coefficients can be included if desired.

Three equation solvers are available for the mechanical energy equation: (1) Gauss-Seidel (iterative), (2) Cholesky decomposition (direct), (3) and Gauss elimination (direct). The analyst may select any of these solvers based on the specific problem; if a specific solver is not selected, SAFSIM attempts to use Gauss-Seidel iteration first. If convergence is not met, or if the coefficient matrix is not diagonally dominant, Cholesky decomposition is employed. This is a direct solver that operates only on terms within the semibandwidth of the coefficient matrix and is therefore relatively fast. If the coefficient matrix is not positive definite, Cholesky will fail and Gauss elimination is used. This is the slowest of the three solvers but the most general. This solver uses partial pivoting and is written so that only the terms within the bandwidth of the coefficient matrix are included, greatly increasing speed. The use of multiple numerical solvers adds to the robustness of the program.

Two solvers are available for solution of the thermal energy equation: (1) Gauss-Seidel, and (2) Gauss elimination. For flow problems that are advectively dominated (such as many gas flow 
problems), Gauss-Seidel provides a rapid solution for the fluid temperature field. Gauss elimination is included for flow problems that are not advectively dominated because the coefficient matrix may lose its diagonal dominance. Cholesky decomposition is not included for the thermal energy solution because the coefficient matrix is not symmetric due to the advective term in the equation.

\section{Structure Heat Transfer}

The structure heat transfer physics module is based on a 1-D finite element model that allows the user to model conduction in any heat transfer structure such as pipe walls, fuel elements or particles, vessel walls, thermocouples, etc. The user can include as many structures as desired. Multiple exchange surfaces allow the user to convectively or radiatively couple any heat transfer structure finite element to any fluid mechanics finite element(s). The module can be run in static or dynamic mode. In dynamic mode, the module automatically determines the optimum implicitness factor for each node of each structure at each time step. Time step size can be automatically calculated for each structure by the program or user specified.

Material properties can be temperature dependent if desired. Also, several options are available for supplying the property data, including tables, polynomials, power laws, and constants. An extensive built-in heat transfer coefficient correlation library includes correlations for laminar and turbulent flows, for internal and external flow geometries, and for gases and liquids (including liquid metals). Temperature, heat flux, and convective/radiative boundary conditions can be specified. Also, finite elements from different heat transfer structures can be conductively (including a contact resistance) or radiatively (including view factors) coupled. This coupling, however, is explicit. A tridiagonal numerical solver provides a rapid solution for the node temperatures of all of the structures.

\section{Reactor Dynamics}

The reactor dynamics physics module is based on a point (0-D) kinetics model with feedback. Multiple reactors can be specified, and multiple feedback coefficients are allowed for each reactor to account for all system interactions. The analyst has complete control over how the feedback coefficients are defined. Also, several "control laws" are available to simulate control rod/drum reactivity control for reactor startup and shutdown simulations. Special-purpose control laws can be added to the program by the analyst if desired. The analyst can specify any number of delayed neutron groups and any number of decay heat groups. A source term also can be included.

Two solvers are currently available for integration of the reactor dynamics equations: (1) Euler, and (2) Runge-Kutta-Fehlberg (RKF). Adaptive time stepping is employed by both solvers based on the desired relative truncation error. The Euler integrator employs step doubling to provide the truncation error estimate for time step selection. The RKF integrator determines an error estimate as the difference between a fourth- and fifth-order prediction. The analyst can switch between solvers during a problem if desired.

\section{Function-Controlled Variables}

In addition to the three physics modules, SAFSIM contains a unique input feature: functioncontrolled variables. This feature allows the analyst to specify many of the input variables as functions of output variables. Examples of input variables include: convergence criteria, flow areas, conduction lengths, feedback coefficients, compressor speed, and boundary conditions. Examples of output variables include: pressure, temperature, velocity, and heat transfer coefficient. For example, the diameter of a fluid mechanics finite element can be specified as a function of a heat transfer finite element temperature to simulate the effect of thermal expansion on the flow field. Functions can be specified as functions of other functions to allow complex logic paths to be included via input. A library of functions is included along with a provision for analysts to easily add their own. Examples of mathematical functions in the library include: cosine, square root, averaging, table lookup, polynomials, and conditional statements. 


\section{SAMPLE APPLICATIONS}

To illustrate the type of simulations that can be performed with SAFSIM, two sample applications are briefly presented. Although both applications are related to the simulation of complex nuclear thermal propulsion systems, it is believed that they demonstrate the basic modeling capbilities of SAFSIM. One system is a proposed ground test reactor for testing nuclear fuel elements and the other is an actual propulsion system built to test a nuclear engine.

\section{Sample Application 1}

Figure 1 shows a schematic of a conceptual reactor for testing particle bed nuclear fuel elements (Powell, 1985). Such a fuel element consists of a bed of power-producing particles packed between porous structures referred to as the hot and cold frit. All structures and coolant paths are included in the SAFSIM input model. This axisymmetric reactor contains various material regions and geometries, requiring many of SAFSIM's modeling features. For example, many of the structures are cooled on more than one surface, requiring multiple exchange surfaces. The particle bed requires use of porous media finite elements. The inlet and outlet plenums of the fuel element require use of distributed flow manifold elements. The nozzle at the exit is modeled with a choked flow boundary element. Internal heat generation due to gamma ray and neu. tron heating is included in all structures. Different heat transfer coefficient correlations are used for the different regions depending on geometry. Various reactor feedback coefficients are defined to account for changing density, temperature, and expansion effects. This is but one example of the type of complex system that can be modeled with SAFSIM.

Steady-state and transient simu-

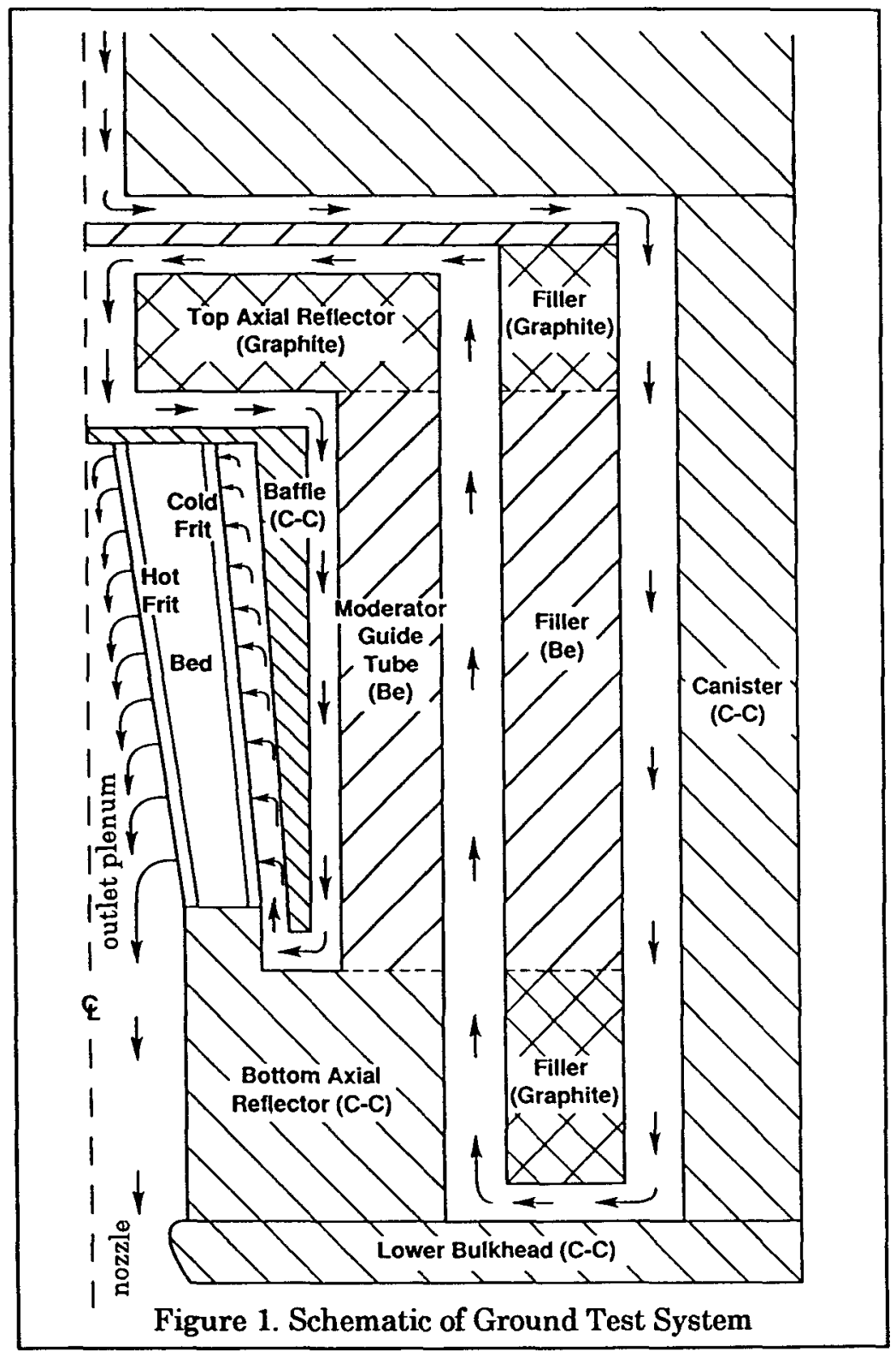
lations of this system were performed to investigate its operating performance. For example, a start. up transient was simulated in which the reactor power was increased five orders of magnitude in $5 \mathrm{~s}$. For this system, running on a $25 \mathrm{MHz} 486 \mathrm{PC}$, a steady-state calculation required on the order of $0.1 \mathrm{~s}$ of computer time per fluid mechanics finite element. Transient calculations required approximately $0.13 \mathrm{~s}$ of computer time per fluid mechanics finite element per simulation second. Thus for a 65 finite element model, a steady-state calculation required $6.5 \mathrm{~s}$ and a $30 \mathrm{~s}$ transient calculation required about 4.2 minutes of execution time. 


\section{Sample Application 2}

Another complex system is a facility built in the 1960 s to test a nuclear rocket engine. This test facility, a corresponding SAFSIM model, and some of the test data are documented in Walton, 1993. Figure 2, extracted from this reference, provides a schematic of the fluid mechanics finite elements used to represent the system. This figure shows the complicated flow splits that must be calculated to simulate the integrated performance of the entire system. For steady state, comparison of predicted versus experimental results indicate very good agreement. Transient calculations are in progress.

\section{CONCLUSIONS}

SAFSIM is a functional computer program that runs on a personal computer or workstation and provides the analyst with a tool for obtaining engineering solutions to complex system analysis problems. SAFSIM development is by no means complete. However, the program architecture was designed with expansion and modification in mind; thus new features and capabilities can be added with relative programming ease. Also, SAFSIM was

designed with many user interfaces built into the program, allowing each analyst to tailor SAFSIM for their particular application without having to fully understand the internal programming structure and solution techniques. Hopefully it is apparent from this paper that versatility is the primary development objective of SAFSIM, making it a candidate analysis tool for a broad range of engineering disciplines. Benchmarking and documentation of the current version are in progress. Additional enhancements are envisioned to make SAFSIM even more versatile, robust, and fast. These planned enhancements will expand the class of problems for which SAFSIM is applicable and are provided in the following non-prioritized list:

(1) LU decomposition solver with iterative refinement for large flow networks

(2) Built-in bandwidth minimizer for the mechanical and thermal energy equation solvers

(3) Turbine finite element

(4) Kagonove solver for the reactor dynamics equations

(5) Structural mechanics physics module

(6) Restart capability

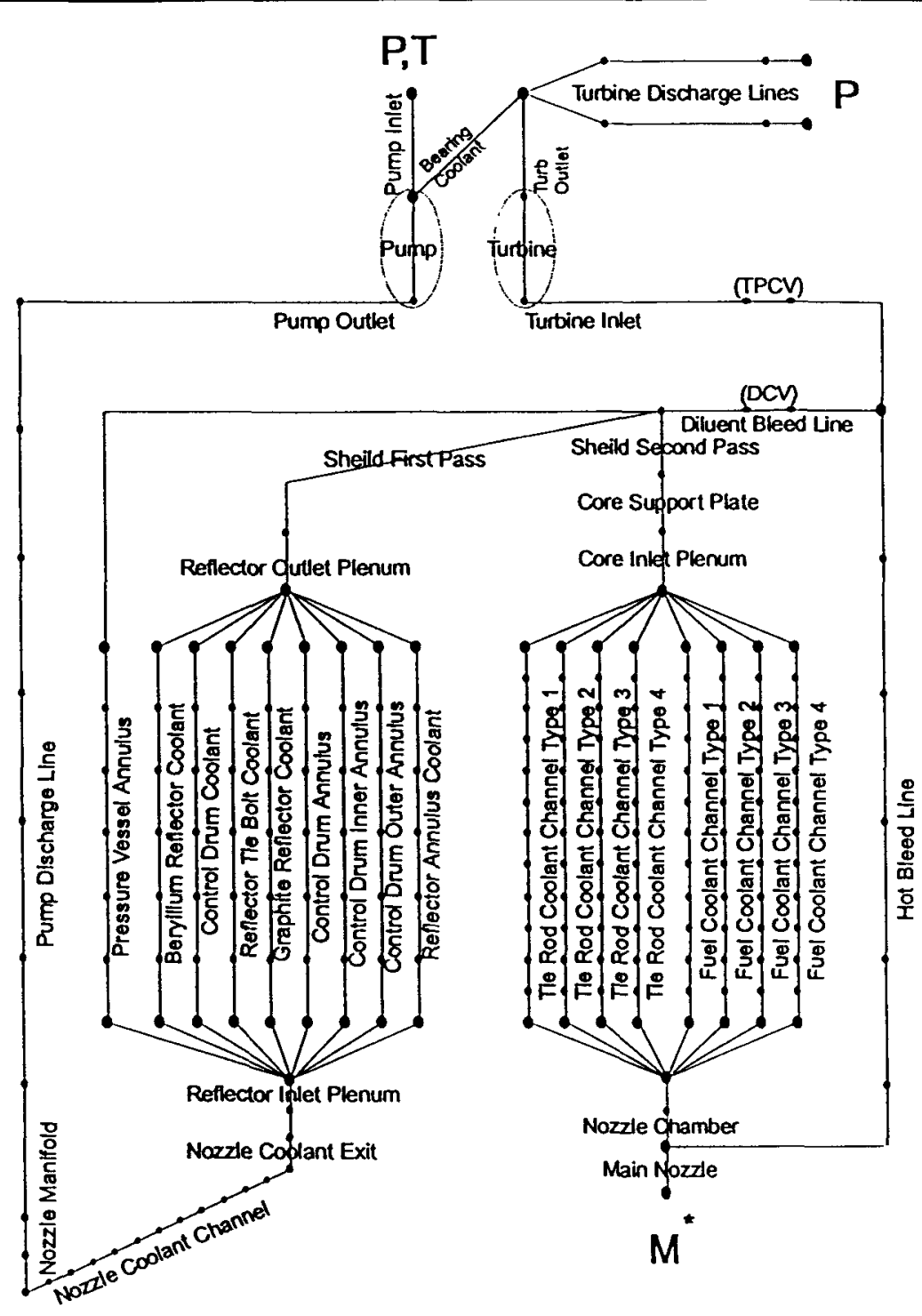

Figure 2. Schematic of SAFSIM Finite Element Model 
(7) Blowdown tank boundary condition option

(8) Generalized source terms for the fluid mechanics equations

(9) 2-D tables and other special functions

(10) Mass accumulation term in the continuity equation

(11) Upwind finite elements for the mechanical energy equation

(12) Liquid metal electromagnetic pump option

(13) Liquid metal accumulator model

(14) Homogeneous equilibrium model for two-phase flow

(15) Pre- and post-processing tools (graphical interface)

\section{Acknowledgments}

This work was funded by the Air Force Space Nuclear Thermal Propulsion program. This work was performed at Sandia National Laboratories, Albuquerque, New Mexico, and was supported by the U. S. Department of Energy under Contract DE-AC04-76DP00789.

\section{References}

Dobranich, Dean, "SAFSIM Input Manual -- A Computer Program for the Engineering Simulation of Flow Systems," Sandia National Laboratories, Albuquerque, NM, SAND92-0694, September 1992.

Powell, J. R., and F. L. Horn, "High Power Density Reactors Based on Direct Cooled Particle Beds," in Space Nuclear Power Systems, M. S. El-Genk and M. D. Hoover, eds., (Malabar, Florida: Orbit Book Company, 1985).

Ransom, V. H., R. J. Wagner, et al, "RELAP5/MOD1 Code Manual Volume 1: System Models and Numerical Methods," NUREG/CR-1826, March 1981.

Walton, James T., "Validation Data For A Nuclear Thermal Propulsion System Model," presented at 29th AIAA/SAE/ ASME/ASEE Joint Propulsion Conference, Paper \# 93-1760, June 28-30, 1993. 